\title{
Efficient Synthesis of Graphene Nanoribbons Sonochemically Cut from Graphene Sheets
}

\author{
Zhong-Shuai Wu, Wencai Ren $(\varangle)$, Libo Gao, Bilu Liu, Jinping Zhao, and Hui-Ming Cheng ( $\varangle)$ \\ Shenyang National Laboratory for Materials Science, Institute of Metal Research, Chinese Academy of Sciences, 72 Wenhua Road, \\ Shenyang 110016, China \\ Received: 23 September 2009 / Revised: 14 November 2009 / Accepted: 17 November 2009 \\ (C) The Author(s) 2010. This article is published with open access at Springerlink.com
}

\begin{abstract}
We report a facile approach to synthesize narrow and long graphene nanoribbons (GNRs) by sonochemically cutting chemically derived graphene sheets (GSs). The yield of GNRs can reach $\sim 5 \mathrm{wt} \%$ of the starting GSs. The resulting GNRs are several micrometers in length, with $\sim 75 \%$ being single-layer, and $\sim 40 \%$ being narrower than $20 \mathrm{~nm}$ in width. A chemical tailoring mechanism involving oxygen-unzipping of GSs under sonochemical conditions is proposed on the basis of experimental observations and previously reported theoretical calculations; it is suggested that the formation and distribution of line faults on graphite oxide and GSs play crucial roles in the formation of GNRs. These results open up the possibilities of the large-scale synthesis and various technological applications of GNRs.
\end{abstract}

\section{KEYWORDS}

Graphene nanoribbon, graphene oxide, synthesis, sonochemical cutting

\section{Introduction}

Graphene nanoribbons (GNRs) are elongated strips of graphene with widths from a few to tens of nanometers which have recently attracted extensive interest because of their unique structure, versatile electronic properties, and promising applications in future carbon-based electronic and spintronic devices [1-6]. Theoretical and experimental studies have both demonstrated that quantum confinement and edge effects make narrow GNRs $(<10 \mathrm{~nm})$ into semiconductors, and the energy gap of GNRs can be easily engineered by varying their width $[1,3]$. This semiconducting nature of narrow GNRs is very different from single-walled carbon nanotubes
(SWCNTs) that contain $\sim 1 / 3$ metallic species, and appears to give them a key advantage over SWCNTs as building blocks for future nanoelectronics. In addition, GNRs are predicted to exhibit a number of other unusual physical phenomena, such as a halfmetallic nature, a spin-polarized ground state in zigzagshaped GNRs and enhanced excitonic effects [4-6]. These attractive properties have stimulated increasing activity in the synthesis of this novel material.

To date, several methods have been developed for the synthesis of GNRs, such as lithographical patterning of graphene [3,7], bottom-up organic synthesis [2], sonochemical cutting of exfoliated expandable graphite [1], chemical vapor deposition $[8,9]$, oxygen plasma etching of graphene using

Address correspondence to Hui-Ming Cheng, cheng@imr.ac.cn; Wencai Ren, wcren@imr.ac.cn 
nanowires as a physical protection mask [10], intercalation/exfoliation [11], and longitudinal unzipping of multi-walled carbon nanotubes (MWCNTs) $[12,13]$. For example, sub-10 nm GNRs have been produced from expandable graphite by a combination of exfoliation and chemical sonication, and field effect transistors based on these GNRs have been shown to exhibit a high on-off ratio of about $10^{7}$ at room temperature [1]. This result opens up the possibility to fabricate narrow GNRs with smooth edges and high device performance, although the yield of GNRs is low [1]. Longitudinal unzipping of MWCNTs $[12,13]$ has recently been shown to produce GNRs in relatively high yield. However, the prepared GNRs usually have a width in excess of $10 \mathrm{~nm}$ due to the limitation imposed by the available diameter of MWCNTs, and exhibit weaker electronic characteristics than GNRs prepared by the sonochemical method. In general, the GNRs synthesized by such methods suffer from rough edges, limited length, or low controllability over the number of layers and the width of GNRs. In particular, it remains a great challenge to produce GNRs with narrow widths and smooth edges in high yield, which has become a bottleneck for further investigation of properties and applicationss of GNRs. Herein, we describe a facile strategy to synthesize GNRs in high yield from chemically derived graphene sheets (GSs). The yield of GNRs is about $5 \mathrm{wt} \%$ of the starting GSs. The resulting GNRs are several micrometers in length, with $\sim 75 \%$ of them being single-layer, and $\sim 40 \%$ being narrower than $20 \mathrm{~nm}$ in width. In addition, a chemical tailoring mechanism of GNRs at the atomic level is proposed based on experimental observations and previously reported theoretical calculations [14-18].

\section{Experimental}

\subsection{Synthesis of GNRs}

Our synthesis method used to prepare GNRs involves two key steps: (1) chemical exfoliation of graphite to prepare GSs, and (2) sonochemically cutting the chemically derived GSs into GNRs. Our previous studies showed that artificial graphite with a low crystallinity and small lateral size is a good choice for preparing single-layer graphene (with $\sim 80 \%$ having a single layer) [19]. Here, we used artificial graphite as the starting material to fabricate GSs via oxidation, thermal exfoliation, $\mathrm{H}_{2}$ reduction, dispersion and centrifugation, as we reported previously [19]. The asprepared GSs $(10 \mathrm{mg})$ were then dispersed in a mixed solution $(100 \mathrm{~mL})$ of $0.1 \mathrm{wt} \%$ polyvinylpyrrolidone (PVP) and $0.1 \mathrm{wt} \%$ sodium dodecyl sulphate to form a stable homogenous suspension by sonication for 1 $\mathrm{h}$ using a high-shear mixer (Goldstar Ultrasonic Instruments, CF1520, $1500 \mathrm{~W}, 20 \mathrm{kHz}$ ). In order to further remove the oxygen-containing groups and to partially restore the extended conjugated $\mathrm{sp}^{2}$ network of GNRs or GSs [20], hydrazine hydrate $(1 \mathrm{~mL})$ was slowly added to the suspension under vigorous stirring, and the resulting solution was refluxed at $100{ }^{\circ} \mathrm{C}$ for $36 \mathrm{~h}$ with a water-cooled condenser. Finally, high-speed centrifugation (12000 rpm for $5 \mathrm{~min}, 3$ times) was used to remove large pieces of GSs and obtain the GNR supernatant.

\subsection{Material characterization}

The morphology and structure of the graphite oxide (GO), GSs, and GNRs were characterized by atomic force microscopy (AFM, Veeco MultiMode NanoScope III a, operating in tapping mode), scanning electron microscopy (SEM, Nova Nano 463, high voltage of $15 \mathrm{kV}$ ), transmission electron microscopy (TEM, JEOL JEM-2010, accelerating voltage of $200 \mathrm{kV}), \quad$ X-ray photoelectron spectroscopy (XPS, Escalab 250, Al K $\alpha$ ), Fourier transform infrared (FTIR) spectroscopy (Nicolet 605XB FTIR spectrometer, KBr disk), and Raman spectroscopy (Jobin Yvon LabRAM HR800, excited by a $632.8 \mathrm{~nm}$ laser with a laser spot size of $\left.\sim 1 \mu \mathrm{m}^{2}\right)$. For Raman measurements, a substrate of $\mathrm{Si}$ capped with $300 \mathrm{~nm}$ thick $\mathrm{SiO}_{2}$ was used to support the GNRs.

\section{Results and discussion}

The as-prepared GNRs were first characterized by AFM, SEM, TEM, and Raman spectroscopy, and the results are shown in Fig. 1. It is important to note from Figs. 1(a), 1(b) and Fig. S-1 in the Electronic Supplementary Material (ESM) that the GNRs have a high density, indicating the effectiveness of our 
synthesis approach. In addition, small GS pieces cut from large GSs and a few large GSs were found in the resulting GNR product. By comparing the masses of the GNRs in the supernatant and the GSs used, it was estimated that this process can produce GNRs in a yield of $\sim 5 \mathrm{wt} \%$ after first centrifugation, which is much higher than yields reported previously $[1,7,21]$. We believe that the yield of GNRs can be further increased by sediment recycling several times. A large number of Raman measurements all showed no evidence of a radial breathing mode in the low-frequency region (100-300 $\left.\mathrm{cm}^{-1}\right)$, as shown in Fig. 1(e), which suggests that the product is not contaminated by SWCNTs. It is interesting to observe two additional peaks at $\sim 1450$ and $1530 \mathrm{~cm}^{-1}$ in the Raman spectra of GNRs, and the origin of these is under investigation in our group. The as-obtained GNRs exhibited good mechanical flexibility and resilience (Fig. S-2 in the ESM), with mechanical bending and folding not leading to any obvious breakage. As a result, the GNRs prepared by our method are much longer than those previously reported in the literature [1], with lengths of up to several micrometers.

Extensive AFM measurements revealed that the topographic height of the GNRs is distributed in the range $0.8-2.2 \mathrm{~nm}$ with three dominant peaks located at $1.1 \mathrm{~nm}$, $1.5 \mathrm{~nm}$, and $1.9 \mathrm{~nm}$ (Fig. 2(a)). These values are in agreement with those reported by Li et al. [1] and correspond to the thicknesses of single-, double- and triple-layer GNRs, respectively. In the product, $\sim 75 \%$ of the GNRs were single-layer, $\sim 15 \%$ double-layer, and $\sim 10 \%$ triple-layer. In addition, $\sim 85 \%$ of GNRs were $5-50 \mathrm{~nm}$ in width with $\sim 40 \%$ of

them being narrower than $20 \mathrm{~nm}$ (Fig. 2(b)). Detailed AFM and TEM observations (Figs. 1(c), 1(d) and Fig. S-3 in the ESM) reveal that most of the GNRs exhibit regular shapes with two parallel smooth edges. However, wedge-shaped GNRs were also occasionally observed with an angle of $10^{\circ}-30^{\circ}$ between two
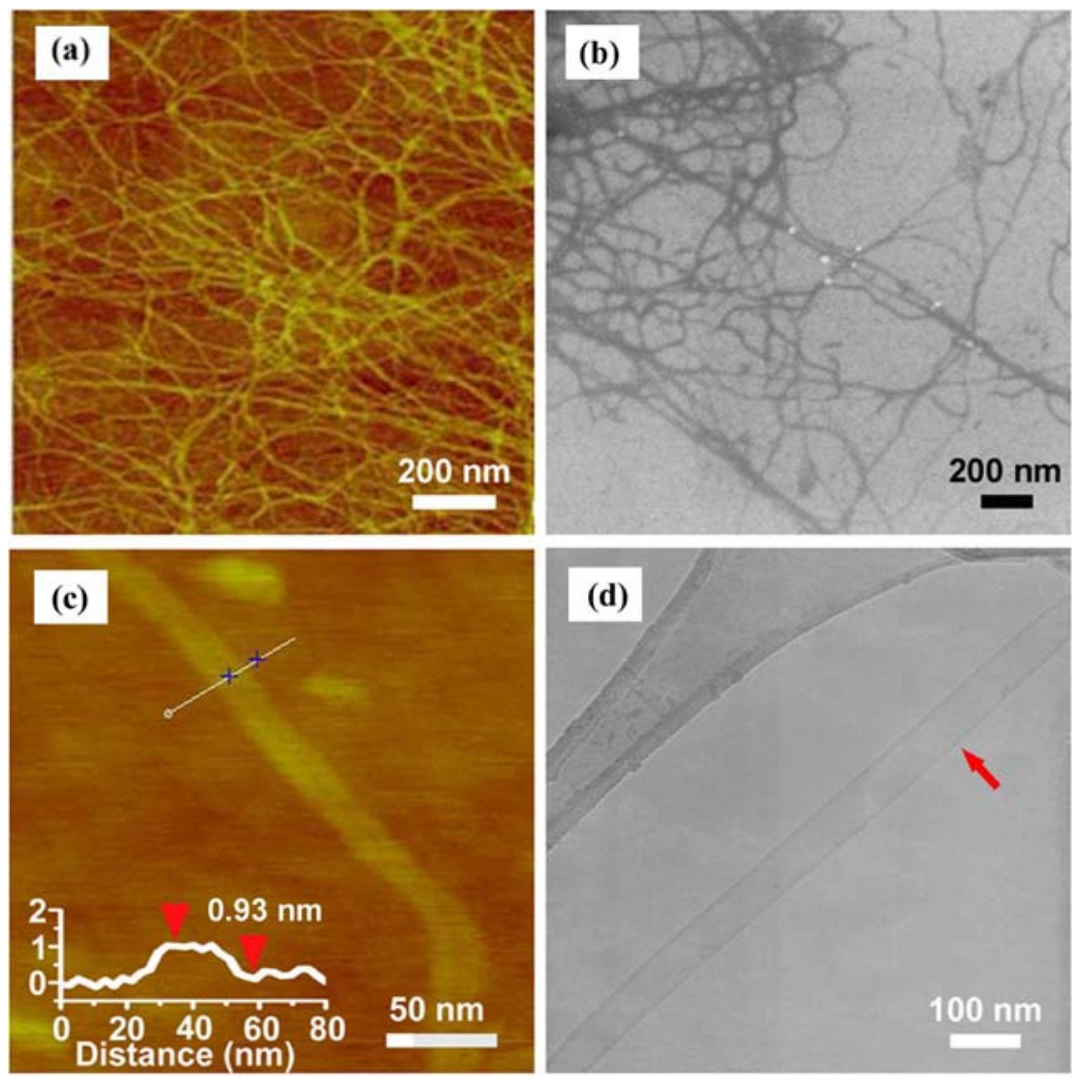

(d)

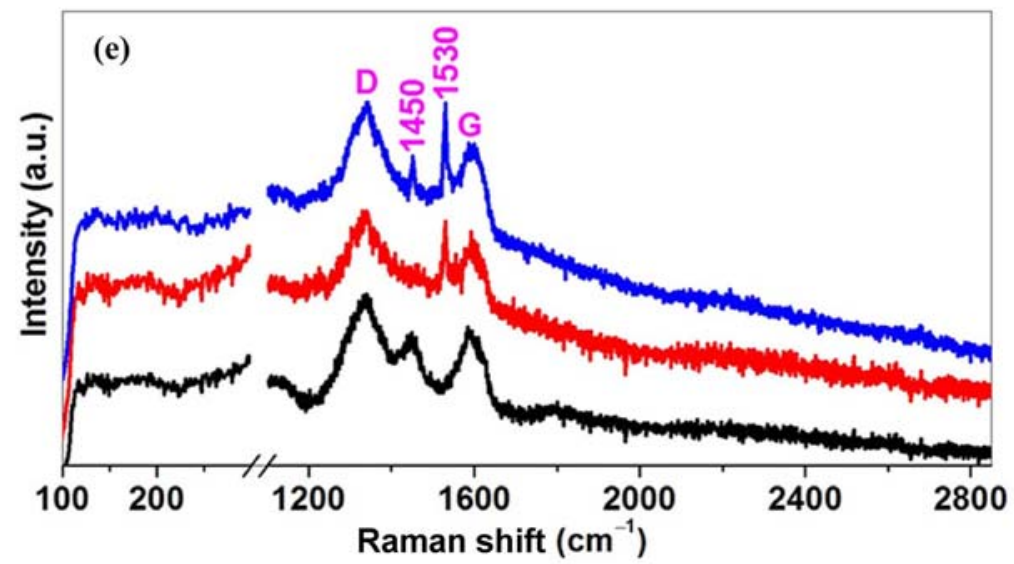

Figure 1 (a) AFM and (b) SEM images of the GNRs spin-coated from the GNR supernatant on a $\mathrm{Si}$ substrate capped with 300-nm-thick $\mathrm{SiO}_{2}$. (c) AFM image of an individual GNR. Inset: the height profile reveals that the measured GNR has a height of $\sim 0.93 \mathrm{~nm}$ and width of $\sim 20 \mathrm{~nm}$. (d) TEM image of a GNR with a width of $\sim 60 \mathrm{~nm}$ (indicated by a red arrow). (e) Typical Raman spectra of the as-prepared GNRs on a $\mathrm{Si}$ substrate capped with $300 \mathrm{~nm}$ thick $\mathrm{SiO}_{2}$ 


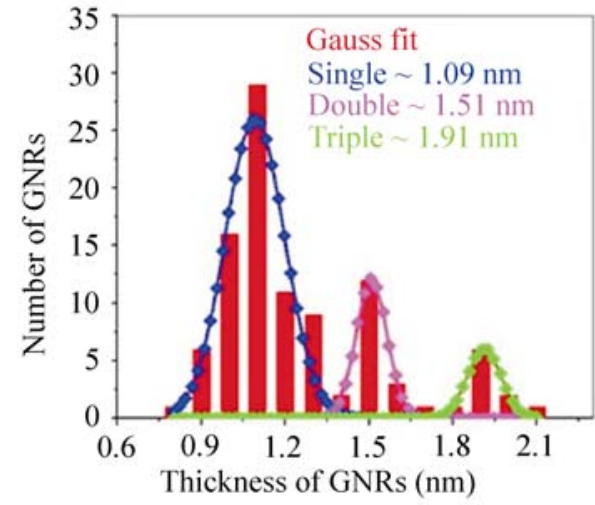

(a)

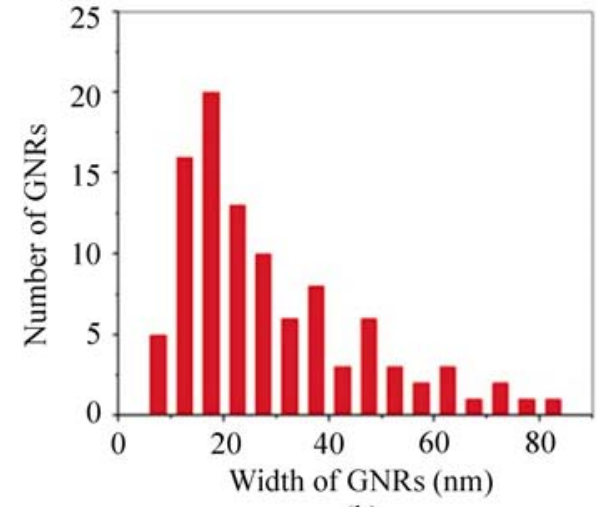

(b)

Figure 2 The thickness (a) and width (b) distributions of 100 individual GNRs

edges (Fig. S-4 in the ESM), which indicates that cutting does not proceed exactly along the crystallographic orientations; consequently the edges of the GNRs may be not identical zigzag or armchair configurations although they look smooth by TEM.

To estimate the quality of the GNRs obtained, we carried out XPS and FTIR measurements. Figure 3(a) shows the XPS of as-prepared GNRs recorded between 0 and $1100 \mathrm{eV}$, which reveals the presence of carbon $(\mathrm{C} 1 \mathrm{~s}, 284.5 \mathrm{eV})$, oxygen $(\mathrm{O} 1 \mathrm{~s}, 532 \mathrm{eV})$, and nitrogen $(\mathrm{N} \mathrm{1s}, 400 \mathrm{eV})$. Based on quantitative analysis of the XPS data, the atomic percentages of $\mathrm{C}$, $\mathrm{O}$, and $\mathrm{N}$ were determined to be $93.57 \%, 6.08 \%$, and $0.35 \%$, respectively. It is worth noting that the $\mathrm{C} / \mathrm{O}$ atomic ratio of the as-prepared GNRs (15.3) is higher than that reported for chemically-derived GSs (12.0)

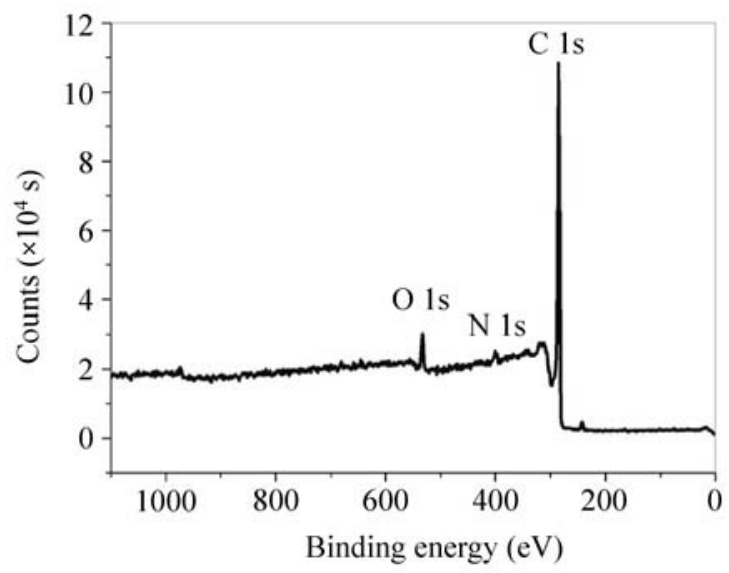

(a)
[19]. In addition, the $\mathrm{C} 1 \mathrm{~s}$ peak of the as-produced GNRs is similar to that of highly oriented pyrolytic graphite (HOPG) (Fig. 3(b)), and no obvious band arising from a carboxylic group at $1730 \mathrm{~cm}^{-1}$ can be observed in the FTIR spectrum (Fig. 4). All these results indicate the effective de-oxygenation and partial restoration of the carbon network of GNRs during the hydrazine reduction. However, a higher intensity ratio of the $\mathrm{D}$ band to the $\mathrm{G}$ band, as well as broadening of the $D$ and $G$ bands were observed, as shown in Fig. 1(e); this can be attributed to a significant contribution from edge atoms of GNRs, the presence of residual oxygen-containing groups in GNRs, and vacancies and topological defects introduced by both decomposition of functional groups and sonication.

In order to determine the key factors affecting the synthesis of GNRs, we performed synthesis experiments with varying parameters and several interesting results were found. (1) Far fewer GNRs were obtained if natural flake graphite was used as the starting material for the synthesis of GSs instead of artificial graphite. (2) An appropriate sonication duration time

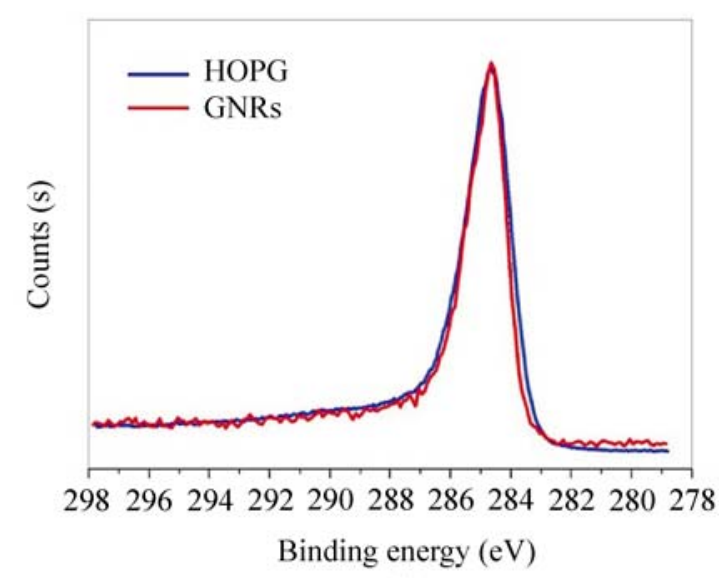

(b)

Figure 3 (a) XPS of GNRs recorded between 0 and $1100 \mathrm{eV}$. (b) The intensity normalized C 1s XPS of GNRs (red line) and pristine HOPG (blue line). The similarity of the spectral features shown in (b) indicates that the GNRs were considerably deoxygenated 
is another key factor for the efficient production of GNRs. Only a small amount of GNRs was obtained with a duration time shorter than $0.5 \mathrm{~h}$, while only small graphene pieces and short GNRs were obtained for very long sonication times ( $\geqslant 10 \mathrm{~h}$, Fig. 5(a)). (3) If the GSs contain a high percentage of oxygen functional groups ( $\mathrm{C} / \mathrm{O}$ ratio $<7$ ), e.g., when no $\mathrm{H}_{2}$ reduction process was employed during the preparation of GSs, the final product consists mostly of small fragments of GSs with only a few GNRs (Fig. 5(b)). (4) As mentioned by Li et al. [1], the use of ambiphilic linear polymers also plays an important role in the fabrication of long GNRs. Strong non-covalent interactions between the ambiphilic polymer (here, PVP) and GSs

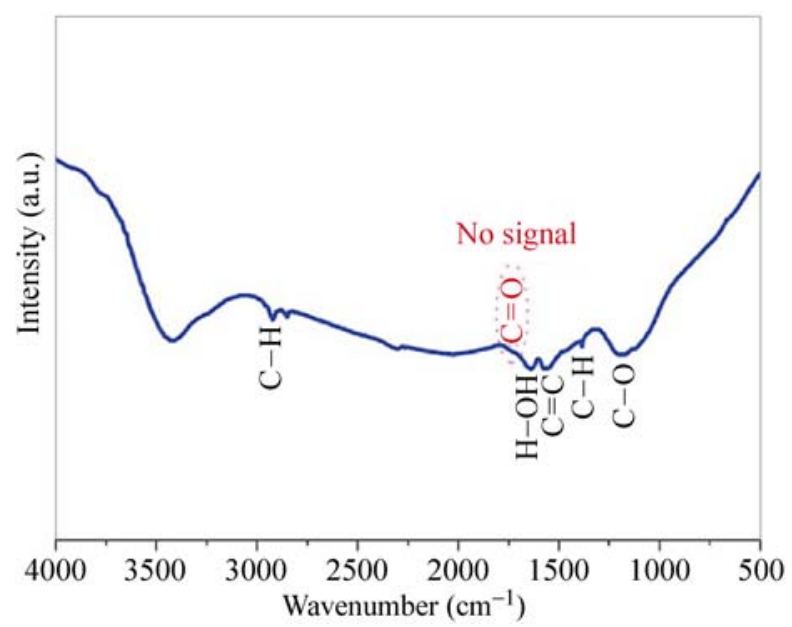

Figure 4 FTIR spectrum of the GNRs. No obvious signal characteristic of a carboxylic group can be observed at $1730 \mathrm{~cm}^{-1}$ (indicated by the dotted ring), indicating considerable deoxygenation of GNRs
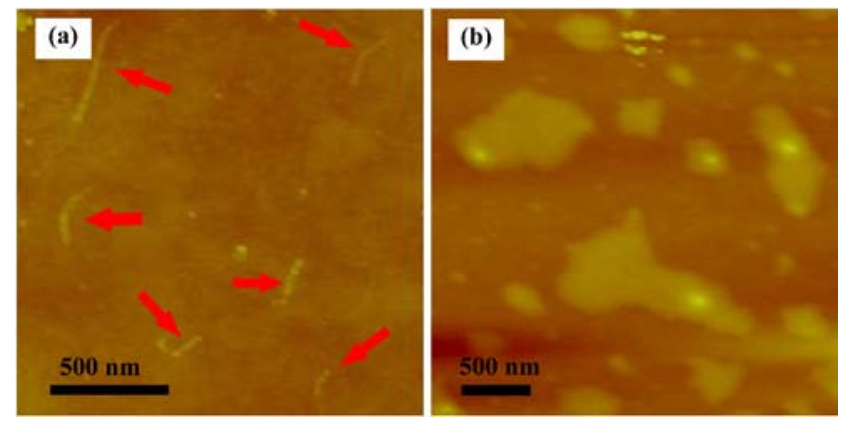

Figure 5 (a) AFM image of short GNRs (indicated by red arrows) produced by sonication treatment for a duration of more than $10 \mathrm{~h}$. (b) AFM image of small graphene pieces obtained from GSs with a high percentage of oxygen-containing groups (C/O: 6.0) decreases the possibilities of cutting long ribbons into shorter ones and short ribbons into smaller pieces during the vigorous sonication process.

In order to elucidate the mechanism of formation of GNRs, we carefully investigated the structure of the GSs and GO by using SEM. It is interesting to find that there are many parallel line faults on their surfaces (Fig. 6 and Figs. S-5, S-6 in the ESM). On the basis of these experimental observations and the previously reported theoretical results [14-18], we propose the following possible mechanism of formation of GNRs: during the oxidation, oxygen atoms bind to the starting graphite material (artificial graphite), and epoxy groups $(\mathrm{C}-\mathrm{O}-\mathrm{C})$ are formed by joining two adjacent carbon atoms. As $\mathrm{Li}$ et al. pointed out, this triangular epoxy ring is severely strained because the two carbon atoms need to undergo configuration changes from a planar $\mathrm{sp}^{2}$-hybridized to a distorted $\mathrm{sp}^{3}$-hybridized geometry [14]. Mechanically, the oxygen atom acts as a minuscule wedge, pushing apart the bridge carbon atoms and stretching the $\mathrm{C}-\mathrm{C}$ bond [15]. More importantly, these epoxy groups do not act individually but rather cooperatively, and they tend to line up on the graphite surface since the side-byside parallel positioning of the epoxy bridges is energetically favored $[14,15]$. This is the reason why many parallel line faults were observed on the GO surface (Fig. 6(a) and Fig. S-5 in the ESM) in our experiments. When GO is heated, some of the functional groups decompose to give $\mathrm{CO}_{2}$ with loss of the van der Waals forces holding the GSs together in GO and leaving behind vacancies and topological defects on the GSs [17]. We believe that the line defects on GSs (Fig. 6(b) and Fig. S-6 in the ESM) act as the initial unzipped sites, due to the interactions there being weaker than normal $\mathrm{C}-\mathrm{C}$ bonds. During the sonication treatment of GSs, the sonochemical effects and ultrahot gas bubbles [1] can break the stretched $\mathrm{C}-\mathrm{C}$ bonds or $\mathrm{C}-\mathrm{O}-\mathrm{C}$ bonds along the line defects (Fig. 6(c)) [18]. If the line faults are parallel, regular GNRs can be formed. Otherwise, wedge-like GNR structures form. Therefore, the location and distribution of line faults on GSs play a key role in the final armchair/zigzag structure of GNRs. The widths and lengths of GNRs are determined by the separation and length of the two adjacent line faults, 

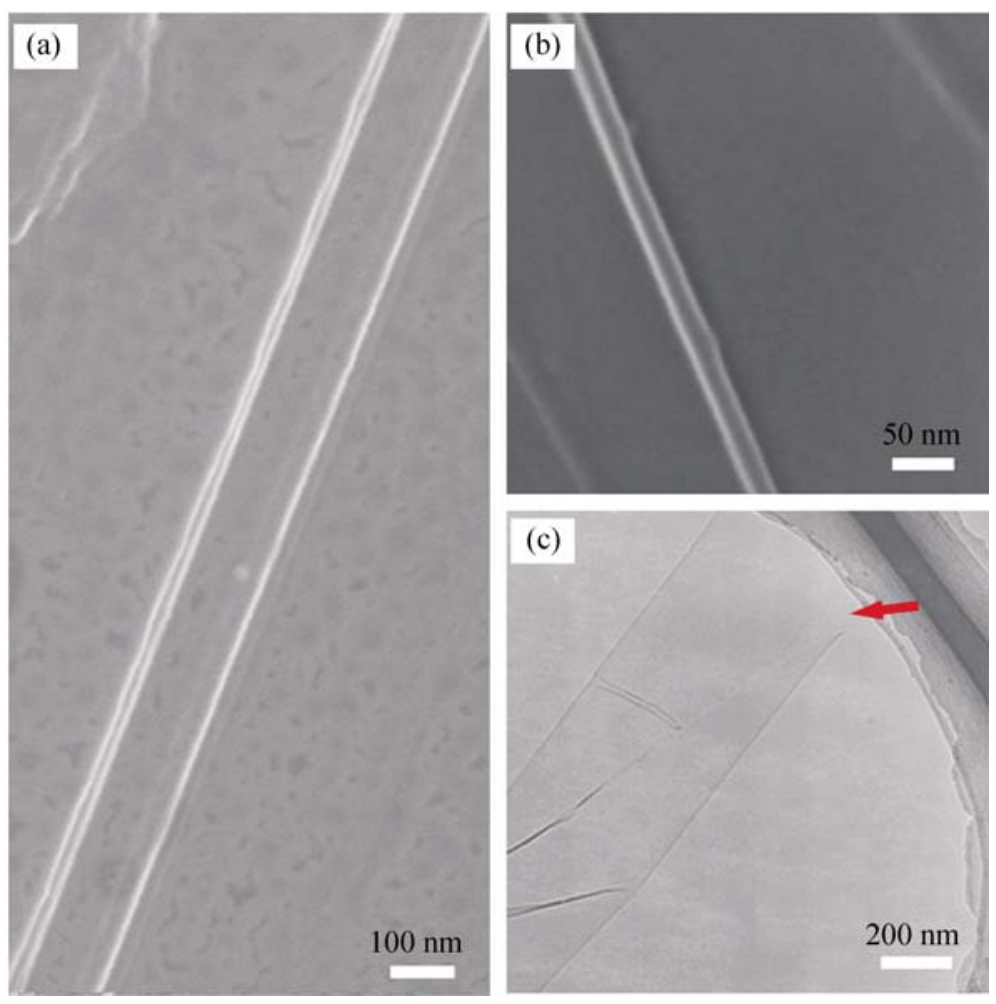

Figure 6 (a) SEM image of three long parallel line faults (white lines) on the GO surface, with separations of several nanometers to tens of nanometers. (b) Two parallel line defects on the GS surface with a separation of $\sim 25 \mathrm{~nm}$. (c) TEM image of the as-prepared GS, showing evidence of cutting GSs along the line fault (indicated by a red arrow)

and the edge structure depends on the direction of the line faults.

\section{Conclusions}

We have developed a facile and effective approach to fabricate GNRs by sonochemically cutting chemically derived GSs. The yield of GNRs can reach $\sim 5 \mathrm{wt} \%$ of the starting GSs. The GNRs are several micrometers in length, with $\sim 75 \%$ of them being single-layer, and $\sim 85 \%$ of them being $5-50 \mathrm{~nm}$ in width. A chemical tailoring mechanism was proposed on the basis of the experimental observations and previously reported theoretical calculations. It is suggested that the formation and distribution of line faults on the surfaces of GO and GSs play important roles in the formation and structure of the GNRs. These results provide valuable information about the controllable and large-scale synthesis of GNRs, and accordingly will accelerate various technological applications of GNRs.

\section{Acknowledgements}

This work was financially supported by the National Natural Science Foundation of China (Nos. 50872136, 50972147, and 50921004), and the Chinese Academy of Sciences (No. KJCX2YW-231). The authors thank Dr. C. Jiang for valuable help on the AFM measurements.

\section{Electronic Supplementary Material:} AFM and TEM characterization of the prepared GNRs, and SEM images of the line defects on the surface of GO and GSs are available in the online version of this article at http: //dx.doi.org/10.1007/s12274$010-1003-7$ and is accessible free of charge.

\section{References}

[1] Li, X. L.; Wang, X. R.; Zhang, L.; Lee, S. W.; Dai, H. J. Chemically derived, ultrasmooth graphene nanoribbon semiconductors. Science 2008, 319, 1229-1232.

[2] Yang, X. Y.; Dou, X.; Rouhanipour, A.; Zhi, L. J.; Rader, H. J.; Mullen, K. Two-dimensional graphene nanoribbons. $J$. Am. Chem. Soc. 2008, 130, 4216-4217.

[3] Han, M. Y.; Ozyilmaz, B.; Zhang, Y. B.; Kim, P. Energy band-gap engineering of graphene nanoribbons. Phys. Rev. Lett. 2007, 98, 206805.

[4] Son, Y. W.; Cohen, M. L.; Louie, S. G. Half-metallic graphene nanoribbons. Nature 2006, 444, 347-349.

[5] Yamashiro, A.; Shimoi, Y.; Harigaya, K.; Wakabayashi, K. Spin- and charge-polarized states in nanographene ribbons with zigzag edges. Phys. Rev. B 2003, 68, 193410.

[6] Yang, L.; Cohen, M. L.; Louie, S. G. Excitonic effects in the optical spectra of graphene nanoribbons. Nano Lett. 2007, 7, 3112-3115.

[7] Tapaszto, L.; Dobrik, G.; Lambin, P.; Biro, L. P. Tailoring the atomic structure of graphene nanoribbons by scanning 
tunnelling microscope lithography. Nat. Nanotechnol. 2008, 3, 397-401.

[8] Campos-Delgado, J.; Romo-Herrera, J. M.; Jia, X. T.; Cullen, D. A.; Muramatsu, H.; Kim, Y. A.; Hayashi, T.; Ren, Z. F.; Smith, D. J.; Okuno, Y. et al. Bulk production of a new form of $\mathrm{sp}^{2}$ carbon: Crystalline graphene nanoribbons. Nano Lett. 2008, 8, 2773-2778.

[9] Wei, D.; Liu, Y.; Zhang, H.; Huang, L.; Wu, B.; Chen, J.; $\mathrm{Yu}, \mathrm{G}$. Scalable synthesis of few-layer graphene ribbons with controlled morphologies by a template method and their applications in nanoelectromechanical switches. J. Am. Chem. Soc. 2009, 131, 11147-11154.

[10] Bai, J.; Duan, X.; Huang, Y. Rational fabrication of graphene nanoribbons using a nanowire etch mask. Nano Lett. 2009, 9, 2083-2087.

[11] Cano-Márquez, A. G.; Rodríguez-Macías, F. J.; CamposDelgado, J.; Espinosa-González, C. G.; Tristán-López, F.; Ramírez-González, D.; Cullen, D. A.; Smith, D. J.; Terrones, M.; Vega-Cantú, Y. I. Ex-MWNTs: Graphene sheets and ribbons produced by lithium intercalation and exfoliation of carbon nanotubes. Nano Lett. 2009, 9, 1527-1533.

[12] Jiao, L.; Zhang, L.; Wang, X.; Diankov, G.; Dai, H. J. Narrow graphene nanoribbons from carbon nanotubes. Nature 2009, 458, 877-880.

[13] Kosynkin, D. V.; Higginbotham, A. L.; Sinitskii, A.; Lomeda, J. R.; Dimiev, A.; Price, B. K.; Tour, J. M. Longitudinal unzipping of carbon nanotubes to form graphene nanoribbons. Nature 2009, 458, 872-877.
[14] Li, J. L.; Kudin, K. N.; McAllister, M. J.; Prud'homme, R. K.; Aksay, I. A.; Car, R. Oxygen-driven unzipping of graphitic materials. Phys. Rev. Lett. 2006, 96, 176101.

[15] Ajayan, P. M.; Yakobson, B. I. Materials science-Oxygen breaks into carbon world. Nature 2006, 441, 818-819.

[16] Li, Z.; Zhang, W.; Luo, Y.; Yang, J.; Hou, J. G. How graphene is cut upon oxidation? J. Am. Chem. Soc. 2009, 131, 6320-6321.

[17] Schniepp, H. C.; Li, J. L.; McAllister, M. J.; Sai, H.; Herrera-Alonso, M.; Adamson, D. H.; Prud'homme, R. K.; Car, R.; Saville, D. A.; Aksay, I. A. Functionalized single graphene sheets derived from splitting graphite oxide. $J$. Phys. Chem. B 2006, 110, 8535-8539.

[18] Kutana, A.; Giapis, K. P. Analytical carbon-oxygen reactive potential. J. Chem. Phys. 2008, 128, 234706.

[19] Wu, Z. S.; Ren, W.; Gao, L.; Liu, B.; Jiang, C.; Cheng, H. M. Synthesis of high-quality graphene with a pre-determined number of layers. Carbon 2009, 47, 493-499.

[20] Stankovich, S.; Dikin, D. A.; Piner, R. D.; Kohlhaas, K. A.; Kleinhammes, A.; Jia, Y.; Wu, Y.; Nguyen, S. T.; Ruoff, R. $\mathrm{S}$. Synthesis of graphene-based nanosheets via chemical reduction of exfoliated graphite oxide. Carbon 2007, 45, 1558-1565.

[21] Valles, C.; Drummond, C.; Saadaoui, H.; Furtado, C. A.; He, M.; Roubeau, O.; Ortolani, L.; Monthioux, M.; Penicaud, A. Solutions of negatively charged graphene sheets and ribbons. J. Am. Chem. Soc. 2008, 130, 15802-15804. 